\title{
Avaliação da amplitude de movimento na Artrite Reumatoide: uma revisão de literatura
}

\author{
Melissa Andrea Jeannet Michaelsen Cardoso Mezzari, Juliane de Oliveira, \\ Susana Cristina Domenech, Noé Gomes Borges Júnior, Monique da Silva Gevaerd
}

Universidade do Estado de Santa Catarina - UDESC, Florianópolis, SC, Brasil.

\begin{abstract}
Resumo: Objetivo: O objetivo desta revisão de literatura é descrever métodos de avaliação de $\mathrm{ADM}$ na AR, a fim de auxiliar na definição das principais limitações apresentadas pelos pacientes e, desta forma, contribuir para um tratamento específico às articulações mais acometidas. Método: A metodologia utilizada foi uma revisão crítica da literatura e a busca foi realizada através do portal da capes, e se restringiu às bases de dados da área da saúde. Além de utilizados os descritores estabelecidos pelo DeCS, "rheumatoid arthritis" e "mobility limitation", inseriu-se na busca o termo "range of motion". Resultados: Na revisão crítica da literatura, foi identificado um total de 774 artigos, dos quais, 446 foram pré-selecionados pelo conteúdo do título e do resumo. Destes, 403 foram excluídos após a leitura, por não preencherem os critérios de inclusão para este estudo. Dessa forma, foram selecionados 43 artigos, sendo estes resumidos de forma padronizada. Os estudos não trazem a ênfase ao estudo da mobilidade articular de indivíduos com AR, mas trazem em comum a inclusão da avaliação da ADM como uma maneira de acompanhar a evolução da doença diretamente à função das articulações, principalmente as mais comprometidas. Conclusão: Sugere-se o estudo completo do comprometimento articular, a fim de traçar propósitos de intervenção ou prevenção de deformidades geradas pela AR, que comprometem a qualidade de vida destes pacientes, e difundir tais práticas de avaliação entre a equipe multidisciplinar que acompanha este perfil, a fim de otimizar o plano de assistência a longo prazo, levando-se em conta a cronicidade da condição.
\end{abstract}

Palavras-chave: Artrite Reumatoide, Limitação da Mobilidade, Amplitude de Movimento Articular.

\section{Motion range assessment in Rheumatoid Arthritis: a literature review}

\begin{abstract}
Objective: The aim of this review is to describe the methods used for range of motion (ROM) assessment in Rheumatoid Arthritis (RA) in order to assist in the identification of the main limitations experienced by patients and thus contribute to the specific treatment of the most affected joints. Method: The methodology used was a critical review of the literature and the search was conducted in the capes portal, specifically in health care databases. The two MeSH descriptors "rheumatoid arthritis" and "mobility limitation" were used in the search, and also the term "range of motion". Results: A total of 774 articles were found in the review and 446 were preselected based on title and abstract contents. After reading, 403 articles did not meet the inclusion criteria set in the study and were excluded. Thus, 43 articles were selected, which are summarized in a standardized manner. The studies do not approach the mobility of joints of RA patients, but they have in common the inclusion of ROM assessment to monitor the disease in a manner that is directly related to the function of the joints, especially the most compromised ones. Conclusion: We suggest the realization of thorough studies on joint involvement in order to outline interventions for preventing deformities generated by RA that compromise the quality of life of these patients. The studies could also contribute to disseminate such assessment practices among the multidisciplinary teams that provide assistance to these patients, optimizing the long-term care plan and taking into account the chronicity of the condition.
\end{abstract}

Keywords: Rheumatoid Arthritis, Mobility Limitation, Joint Range of Motion. 


\section{Introdução}

A Artrite Reumatoide (AR) é uma doença inflamatória crônica, associada a alterações imunológicas sistêmicas que afeta de forma simétrica as articulaçôes sinoviais, com predileçẫo por articulaçôes periféricas (SANTANA et al., 2014; OLIVEIRA et al., 2015; NISHIKAWA et al., 2015). Acomete $0,5 \%$ a $1 \%$ da população mundial (HAMBRIGHT et al., 2011; FALEIRO et al., 2011). No Brasil, os estudos realizados por Marques Neto et al. (1993) e Senna et al. (2004), encontraram prevalências compatíveis com as estimativas mundiais - $0,2 \%$ a $1 \%$ e $0,46 \%$ de indivíduos com $A R$, respectivamente. $\mathrm{O}$ sexo feminino é duas a três vezes mais afetado pela $A R$, em comparação ao sexo masculino, sendo incomum em homens jovens ( 0 a $0,5 \%)$. A AR pode ocorrer em qualquer faixa etária, apesar de a prevalência aumentar com a idade, geralmente com pico entre a $4^{\mathrm{a}}$ e $6^{\mathrm{a}}$ décadas. Acomete todas as raças e partes do mundo, sem diferença na prevalência quanto à latitude, longitude ou clima (BRANDÁO, 1997; OLIVEIRA et al., 2014).

Os sintomas da AR são decorrentes do quadro inflamatório prolongado e da destruição das articulaçôes, causando dor, rigidez e edema, com consequente perda da força muscular e da amplitude de movimento (ADM) articular (SILVA et al., 2003; SANTANA et al., 2014; OLIVEIRA et al., 2015; NISHIKAWA et al., 2015). Estes sintomas são marcados por períodos de remissão e exacerbação, com principal acometimento nas articulaçóes dos punhos, metacarpofalangeanas, interfalangeanas proximais, metatarsofalangeanas, ombros e joelhos (TORQUETTI et al., 2008). A deformidade das mãos é uma característica típica da $\mathrm{AR}$, bem como em outras articulaçóes, devido ao comprometimento de tendóes, cápsulas, ossos e ligamentos (SWEENEY; FIRESTEIN, 2004). Além do envolvimento articular, manifestaçóes extra-articulares sistêmicas como febre, perda de peso, anemia e náuseas, também são observadas nos pacientes com AR (BERTOLO, 2008). Consequentemente, a AR gera uma reduçáo na capacidade funcional, a qual pode evoluir de forma branda ou severa, prejudicando o desempenho das atividades cotidianas, causando significativo impacto na qualidade de vida dos pacientes, além de mortalidade prematura (ALETAHA et al., 2010).

Vale ressaltar que, devido aos mecanismos de envelhecimento humano, os sistemas do corpo sofrem mudanças, resultando na perda progressiva de funcionalidade relativa ao sistema musculoesquelético (MASANES et al., 2012). Mesmo assim, embora a perda da capacidade funcional em indivíduos hígidos após a idade de 50 anos gire em torno de $10 \%$ (BENDTSEN et al., 1995), estima-se em 20\% a taxa de perda de capacidade funcional na AR (OLIVEIRA et al., 2015). Sendo assim, as consequências negativas da doença em relação às funçóes físicas dos pacientes, as quais são multidimensionais $\mathrm{e}$ envolvem diminuiçáo significativa da força e da ADM, ressaltam a necessidade da realizaçáo de avaliações quantitativas objetivas e confiáveis para o acompanhamento do tratamento multidisciplinar da AR.

Muito se tem pesquisado em avaliação clínica de pacientes com AR e a resposta aos diversos tratamentos, como indicadores clinimétricos e questionários autorrelatados, que avaliam a capacidade funcional e a qualidade de vida (CORBACHO; DAPUETO, 2010). Mas poucos estudos exploram a ADM como parte da avaliaçáo e acompanhamento clínico e em até quanto o nível de atividade da doença interfere nestas medidas funcionais, essenciais para a realização de tarefas do dia a dia. Apesar de se postular que a queixa álgica, rigidez, diminuição de força muscular, restrição da $\mathrm{ADM}$ e deformidades comprometam a funcionalidade na AR (SANTANA et al., 2014; FALEIRO et al., 2011), a avaliação da ADM ainda não faz parte da rotina de acompanhamento do decurso e prognóstico da doença. De acordo com Oliveira et al. (2014), a ADM articular está relacionada à funcionalidade e constitui um fator determinante de morbidade e preditor de mortalidade em pacientes com AR.

Portanto, o objetivo desta revisão de literatura é descrever, de forma crítica, métodos de avaliação de ADM na AR, a fim de auxiliar na definição das principais limitaçóes apresentadas pelos pacientes e, desta forma, contribuir para um tratamento mais específico às articulações mais acometidas, bem como orientar o tipo de atividade a ser evitada, a fim de sugerir mudanças de hábitos da vida diária ou de atividade laboral.

\section{Método}

Esta revisão bibliográfica foi dividida em duas etapas: a primeira etapa referente à procura de descritores estabelecidos pelo DeCS (Descritores em Ciências da Saúde) da Biblioteca Virtual em Saúde. Posteriormente, foram estabelecidos dois critérios para refinar os resultados; a abrangência temporal dos estudos, definida pelas publicaçóes dos últimos 20 anos, e idioma, no qual foram selecionados artigos em inglês e português. 
A busca foi realizada através do portal da capes (COORDENAÇÃO..., 2016) e se restringiu às bases de dados da área da saúde. Não foram encontrados descritores relacionados ao termo "range of motion". Desta forma, além de utilizados os descritores estabelecidos pelo DeCS "rheumatoid arthristis" e "mobility limitation", inseriu-se na busca o termo "range of motion", da seguinte forma: "Arthritis Rheumatoid" AND "Range of Motion"; "Arthritis Rheumatoid AND Mobility Limitation".

Para inclusão dos estudos, considerou-se o seguinte critério: artigos científicos que analisassem a mobilidade articular de indivíduos com AR.

Foram excluídos capítulos de livros, teses e dissertaçóes, assim como a literatura não escrita em língua portuguesa ou inglesa, bem como pesquisas que avaliassem a mobilidade articular em modelos animais.

Para evitar a inclusão de artigos repetidos, os títulos dos artigos encontrados foram tabulados em planilha do Excel (Microsoft Office, 2008). Foram excluídas as referências semelhantes, evitando um número superestimado de trabalhos identificados na busca total, assim se obtendo uma soma real quanto ao número de publicaçóes sobre o tema.

A seleçáo dos estudos foi realizada por dois avaliadores, simultaneamente e de forma independente, seguindo o critério de inclusão pré-estabelecido. Os trabalhos que não apresentaram informaçóes suficientes para exclusão pela leitura do título ou resumo foram lidos na íntegra. As divergências quanto à seleção do material foram discutidas até se alcançar um consenso. A extraçáo dos dados foi realizada seguindo uma ficha padronizada com as seguintes informaçôes: autor e ano de publicação, objetivo, amostra e protocolo de avaliação da ADM.

\section{Resultados}

$\mathrm{Na}$ revisão crítica da literatura, foi identificado um total de 774 artigos, dos quais, 446 foram pré-selecionados pelo conteúdo do título e do resumo. Destes, 403 foram excluídos após a leitura, por não preencherem os critérios de inclusão para este estudo. Dessa forma, foram selecionados 43 artigos, sendo estes resumidos de forma padronizada (Tabela 1).

Tabela 1. Protocolos de avaliação da ADM.

\begin{tabular}{|c|c|c|}
\hline $\begin{array}{l}\text { Autor e ano de } \\
\text { publicação }\end{array}$ & Amostra & Protocolo de avaliação da ADM \\
\hline Lamb et al. (2015) & $\begin{array}{l}\text { Tamanho: } 490 \\
\text { Idade: } 63,5(11) \text { anos. }\end{array}$ & Sem descrição do instrumento/protocolo \\
\hline Oliveira et al. (2015) & $\begin{array}{l}\text { Tamanho: } 32 \\
\text { Idade: } 53 \text { (13) anos. }\end{array}$ & $\begin{array}{l}\text { Escala EPM-ROM (Escola Paulista de } \\
\text { Medicina - Range of Motion); } \\
\text { Goniometria. }\end{array}$ \\
\hline Nishikawa et al. (2015) & $\begin{array}{l}\text { Tamanho: } 30 \\
\text { Idade: } 59,9 \text { anos }\end{array}$ & Sem descrição do instrumento/protocolo. \\
\hline Oliveira et al. (2014) & $\begin{array}{l}\text { Tamanho: } 30 \\
\text { Idade: } 23-65 \text { anos. }\end{array}$ & $\begin{array}{l}\text { Escala EPM-ROM (Escola Paulista de } \\
\text { Medicina - Range of Motion); } \\
\text { Goniometria }\end{array}$ \\
\hline Hooper et al. (2014) & $\begin{array}{l}\text { Tamanho: } 156 \\
\text { Idade: Grupo controle-20-65 } \\
\text { anos; Grupo OA-53-80 anos; } \\
\text { Grupo AR-28-89 anos. }\end{array}$ & $\begin{array}{l}\text { Classificação ADM por escores (autores do } \\
\text { estudo): } 0 \text { - Total; } \\
1 \text { - Limitado; } 2 \text { - Rígido. }\end{array}$ \\
\hline Santana et al. (2014) & $\begin{array}{l}\text { Não se aplica (Revisão de } \\
\text { Literatura). } \\
\text { Tamanho: } 35\end{array}$ & Sentar e alcançar. \\
\hline Sugiura et al. (2014) & $\begin{array}{l}\text { Idade: Grupo AR - 42-77 anos; } \\
\text { Grupo Espondilose }-57-82\end{array}$ & Análise Cinemática. \\
\hline Poole et al. (2013) & $\begin{array}{l}\text { Tamanho: } 156 \\
\text { Idade: DM - 58,6 (15,8); ES - } \\
54,5(10,9) ; \mathrm{AR}-49,2(13,5) ; \mathrm{OA} \\
-62,9(10,1) .\end{array}$ & $\begin{array}{l}\text { Teste Funcional Keitel (KFT) e versão } \\
\text { condensada do Teste de Mobilidade da Mão } \\
\text { (HAMIS). }\end{array}$ \\
\hline $\begin{array}{l}\text { Slungaard e Mengshoel } \\
\text { (2013) }\end{array}$ & $\begin{array}{l}\text { Tamanho: } 123 \\
\text { Idade: } 61(13) \text { anos. }\end{array}$ & $\begin{array}{l}\text { Goniometria. } \\
\text { Protocolo de avaliação da ADMNorkins e } \\
\text { AAOS. }\end{array}$ \\
\hline Dubbeldam et al. (2013) & $\begin{array}{l}\text { Tamanho: } 25 \\
\text { Idade: } 23-78 \text { anos. }\end{array}$ & Análise Cinemática. \\
\hline
\end{tabular}


Tabela 1. Continuação...

\begin{tabular}{|c|c|c|}
\hline $\begin{array}{c}\text { Autor e ano de } \\
\text { publicação }\end{array}$ & Amostra & Protocolo de avaliação da ADM \\
\hline Fulfaro et al. (2012) & $\begin{array}{l}\text { Tamanho: } 12 \\
\text { Idade: } 60-79 \text { anos. }\end{array}$ & Goniômetro computadorizado Biometrics ${ }^{\circledR}$ \\
\hline Lester et al. (2012) & $\begin{array}{l}\text { Tamanho: } 40 \\
\text { Média: Adultos jovens - 21-30; } \\
\text { Adultos maduros - 41-64; AR } \\
\text { sem cirurgia - 40-71; AR pós- } \\
\text { cirurgia - } 48-88 \text {. }\end{array}$ & Análise Cinemática. \\
\hline Dubbeldam et al. (2011) & $\begin{array}{l}\text { Tamanho: } 35 \text {. } \\
\text { Idade: } \mathrm{AR}-46,6(12,8) \\
\text { Saudáveis }-41,6(8,5)\end{array}$ & Análise Cinemática. \\
\hline Niedermann et al. (2011) & $\begin{array}{l}\text { Tamanho: } 53 \\
\text { Idade: } 53,44(15,71)\end{array}$ & Goniometria. \\
\hline Hayashi e Uchiya (2011) & $\begin{array}{l}\text { Tamanho: estudo de caso } \\
\text { Idade: não relatado }\end{array}$ & Goniometria. \\
\hline Nolte et al. (2011) & $\begin{array}{l}\text { Tamanho: } 10 \\
\text { Idade: } 54,1(8,1) \text { anos. }\end{array}$ & Goniometria. \\
\hline Jain et al. (2010) & $\begin{array}{l}\text { Tamanho: } 23 \\
\text { Idade: } 38-87 \text { anos }(\text { média }=58,6)\end{array}$ & Goniometria. \\
\hline Kuhlow et al. (2010) & $\begin{array}{l}\text { Tamanho: } 239 \\
\text { Idade: } 56(13)\end{array}$ & $\begin{array}{l}\text { Escala EPM-ROM (Escola Paulista de } \\
\text { Medicina-Range of Motion); } \\
\text { Goniometria. }\end{array}$ \\
\hline Takatori et al. (2010) & $\begin{array}{l}\text { Tamanho: } 24 \\
\text { Idade: } 43-84 \text { anos. }\end{array}$ & Análise Cinemática. \\
\hline Dias et al. (2009) & $\begin{array}{l}\text { Tamanho: } 54 \\
\text { Idade: } 29-79 \text { anos. }\end{array}$ & "Working space of the hand" \\
\hline $\begin{array}{l}\text { Johnsson e Eberhardt } \\
(2009)\end{array}$ & $\begin{array}{l}\text { Tamanho: } 183 \\
\text { Idade: } 51,4(12,4)\end{array}$ & $\begin{array}{l}\text { Teste de Performance. } \\
\text { Goniometria. }\end{array}$ \\
\hline Tägil et al. (2009) & $\begin{array}{l}\text { Tamanho: } 30 \\
\text { Idade: } 33-69 \text { anos. }\end{array}$ & Análise radiográfica para cálculo da ADM. \\
\hline Brodin et al. (2008) & $\begin{array}{l}\text { Tamanho: } 228 \\
\text { Idade: Intervenção - } 54(14) \text {; } \\
\text { Controle - } 56(13,9)\end{array}$ & $\begin{array}{l}\text { Escala EPM-ROM (Escola Paulista de } \\
\text { Medicina-Range of Motion); } \\
\text { Goniometria. }\end{array}$ \\
\hline Oosterveld et al. (2009) & $\begin{array}{l}\text { Tamanho: } 38 \\
\text { Idade: } 60,5(13,0)\end{array}$ & $\begin{array}{l}\text { Escala EPM-ROM (Escola Paulista de } \\
\text { Medicina - Range of Motion); } \\
\text { Goniometria. }\end{array}$ \\
\hline Bulthuis et al. (2007) & $\begin{array}{l}\text { Tamanho: } 54 \\
\text { Idade: } 29-79 \text { anos. }\end{array}$ & $\begin{array}{l}\text { Escala EPM-ROM (Escola Paulista de } \\
\text { Medicina - Range of Motion); } \\
\text { Goniometria. }\end{array}$ \\
\hline Goodson et al. (2007) & $\begin{array}{l}\text { Tamanho: } 183 \\
\text { Idade: } 51,4(12,4)\end{array}$ & Goniometria. \\
\hline Vermeulen et al. (2006) & $\begin{array}{l}\text { Tamanho: } 30 \\
\text { Idade: } 33-69 \text { anos. }\end{array}$ & Goniometria com nível de precisão de $5^{\circ}$. \\
\hline Valle et al. (2006) & $\begin{array}{l}\text { Tamanho: } 228 \\
\text { Idade: Intervenção - } 54 \text { (14); } \\
\text { Controle - } 56(13,9)\end{array}$ & Teste do Pêndulo de Wartenberg. \\
\hline Häkkinen et al. (2005a) & $\begin{array}{l}\text { Tamanho: } 38 \\
\text { Idade: } 60,5(13,0)\end{array}$ & Análise Cinemática \\
\hline Häkkinen et al. (2005b) & $\begin{array}{l}\text { Tamanho: } 304 \\
\text { Idade: } 21-83 \text { anos. }\end{array}$ & Goniometria com nível de precisão de $5^{\circ}$. \\
\hline $\begin{array}{l}\text { Lefevre-Colau et al. } \\
(2003)\end{array}$ & $\begin{array}{l}\text { Tamanho: } 42 \text { pacientes para o } \\
\text { estudo de confiabilidade (grupo } 1 \text { ). } \\
50 \text { pacientes para o estudo de } \\
\text { validade (grupo } 2 \text { ). } \\
\text { Idade: Grupo } 1 \text { - } 22 \text { a } 80 \text { anos; } \\
\text { Grupo } 2 \text { - } 19 \text { a } 77 \text { anos. }\end{array}$ & Goniometria. \\
\hline
\end{tabular}


Tabela 1. Continuação...

\begin{tabular}{|c|c|c|}
\hline $\begin{array}{l}\text { Autor e ano de } \\
\text { publicação }\end{array}$ & Amostra & Protocolo de avaliação da ADM \\
\hline Meireles et al. (2002) & $\begin{array}{l}\text { Tamanho: } 100 \\
\text { Idade: Grupo controle - } \\
\text { 50,02/22-77 (21,5); Grupo AR - } \\
\text { 50,24/22,78 }(12,3)\end{array}$ & $\begin{array}{l}\text { Escala EPM-ROM (Escola Paulista de } \\
\text { Medicina - Range of Motion); } \\
\text { Goniometria. }\end{array}$ \\
\hline Orces et al. (2002) & $\begin{array}{l}\text { Tamanho: } 273 \\
\text { Idade: } 23-90 \text { anos }\end{array}$ & $\begin{array}{l}\text { Critérios dos autores - ADM normal e ADM } \\
\text { anormal. }\end{array}$ \\
\hline Johnson et al. (2002) & $\begin{array}{l}\text { Tamanho: } 103 \\
\text { Idade: } 34-79 \text { anos. }\end{array}$ & Índice de lesão articular. \\
\hline Fowler e Nicol (2001) & $\begin{array}{l}\text { Tamanho: } 16 \\
\text { Idade: } 31-68 \text { anos. }\end{array}$ & Análise Cinemática. \\
\hline $\begin{array}{l}\text { Hammond e Freeman } \\
\text { (2001) }\end{array}$ & $\begin{array}{l}\text { Tamanho: } 127 \\
\text { Idade: Grupo padrão }-51,56 \\
(9,73) \text {; } \\
\text { Grupo proteção articular }-49,49 \\
(11,43)\end{array}$ & Goniometria. \\
\hline $\begin{array}{l}\text { Van den Ende et al. } \\
\text { (2000) }\end{array}$ & $\begin{array}{l}\text { Tamanho: } 64 \\
\text { Idade: } 60 \text { (13) anos. }\end{array}$ & $\begin{array}{l}\text { Escala EPM-ROM (Escola Paulista de } \\
\text { Medicina - Range of Motion); } \\
\text { Goniometria. }\end{array}$ \\
\hline Cranney et al. (1999) & $\begin{array}{l}\text { Tamanho: } 40 \\
\text { Idade: } 37-82 \text { anos. }\end{array}$ & $\begin{array}{l}\text { Escala de Movimento e Alinhamento } \\
\text { Articular. } \\
\text { Índice de Deformidade - total de articulações } \\
\text { lesionadas. Adaptado de duas escalas: JAM } \\
\text { (Joint Alignment and Motion Scale) e EPM- } \\
\text { ROM (Escola Paulista de Medicina - Range } \\
\text { of Motion). }\end{array}$ \\
\hline Stenström e Nisell (1997) & $\begin{array}{l}\text { Não se aplica (Revisão de } \\
\text { Literatura). }\end{array}$ & $\begin{array}{l}\text { Escala EPM-ROM (Escola Paulista de } \\
\text { Medicina - Range of Motion); } \\
\text { Goniometria. }\end{array}$ \\
\hline $\begin{array}{l}\text { Van den Ende et al. } \\
\text { (1996) }\end{array}$ & $\begin{array}{l}\text { Tamanho: } 100 \\
\text { Idade: Grupo de alta intensidade } \\
\text { - 51,1 }(9,5) \text {; Grupo de baixa } \\
\text { intensidade }-47,7(13,6) ; \\
\text { Baixa intensidade individual - } \\
53,1(12,1) \text {; Exercício de casa } \\
\text { individual - 56,1 }(10,9) \text {. }\end{array}$ & $\begin{array}{l}\text { Escala EPM-ROM (Escola Paulista de } \\
\text { Medicina - Range of Motion); } \\
\text { Goniometria. }\end{array}$ \\
\hline Vlieland et al. (1993) & $\begin{array}{l}\text { Tamanho: } 50 \\
\text { Idade: } 62,1(14,1)\end{array}$ & $\begin{array}{l}\text { Escala EPM-ROM (Escola Paulista de } \\
\text { Medicina - Range of Motion); } \\
\text { Goniometria. }\end{array}$ \\
\hline Ferraz et al. (1990) & $\begin{array}{l}\text { Tamanho: } 35 \\
\text { Idade: } \mathrm{x}=49,2(\mathrm{DP}=13,4) \text { anos. }\end{array}$ & $\begin{array}{l}\text { Escala EPM-ROM (Escola Paulista de } \\
\text { Medicina - Range of Motion); } \\
\text { Goniometria. }\end{array}$ \\
\hline Badley et al. (1984) & $\begin{array}{l}\text { Tamanho: } 95 \\
\text { Idade: } 28-84 \text { anos. }\end{array}$ & Goniometria. \\
\hline
\end{tabular}

\section{Discussão}

A ADM é definida por Floyd (2011) como sendo a área pela qual uma articulação pode se movimentar normalmente de forma livre e indolor. Este conceito pode ser complementado pela definição trazida pelos autores Hamill e Knutzen (1999), Enoka (1999), Kendall e McCreary (1990), como sendo o deslocamento angular de uma articulação, influenciado pelos ligamentos, comprimento músculo-tendíneo e tecidos moles. As ADMs variam de pessoa para pessoa, pois dependem de suas atividades diárias, práticas de atividades físicas, idade, sexo e hereditariedade (HAMILL; KNUTZEN, 1999; ENOKA, 1999).

A ênfase nos estudos que envolvem a avaliação da ADM na clínica reumatológica, em especial a AR, ainda é de cunho ortopédico, principalmente para avaliar intervenções cirúrgicas (NISHIKAWA et al., 2015; LESTER et al., 2012; HAYASHI; UCHIYA, 2011; JAIN et al., 2010; TÄGIL et al., 2009) ou 
avaliaçóes cinemáticas (SUGIURA et al., 2014; DUBBELDAM et al., 2013). Poucos estudos contemplam a avaliação da ADM global como destaque das avaliaçóes funcionais. Isto parece um contrassenso, tendo em vista o caráter de restrição articular de a AR estar diretamente relacionada à prática das AVDs e ser táo importante quanto a dinamopenia e a sarcopenia muscular para o desempenho de atividades laborais e não laborais.

Neste sentido, a avaliação da amplitude de movimento (ADM), que já é extensamente utilizada para quantificar o déficit musculoesquelético em outras disfunçóes, principalmente as de origem ortopédica, quando aliada à avaliação da capacidade funcional, pode verificar em que nível as doenças ou agravos impedem o desempenho das atividades cotidianas de forma autônoma e independente, ou seja, sem a necessidade de adaptaçóes ou de auxílio de outras pessoas, permitindo o desenvolvimento de um planejamento assistencial mais adequado (DUARTE et al., 2007). Essa avaliação se torna, portanto, essencial para estabelecer diagnóstico, prognóstico e julgamento clínico adequados, que servirão de base para as decisôes sobre os tratamentos e cuidados necessários da equipe multidisciplinar que atua no indivíduo com AR. É um parâmetro que, associado a outros indicadores de saúde, pode ser utilizado para determinar a eficácia e a eficiência das intervençôes propostas.

A medida da ADM articular é um componente importante na avaliação física, pois identifica as limitaçôes articulares, bem como permite aos profissionais de saúde acompanhar de modo quantitativo a eficácia das intervençôes terapêuticas durante a reabilitação. Gajdosik e Bohannon (1987) afirmam que as avaliaçôes goniométricas são uma excelente ferramenta para que os fisioterapeutas quantifiquem os níveis de limitação de movimento e, desta forma, decidam por intervençôes terapêuticas apropriadas e a documentação da efetividade dessas. Essas medidas são rotineiramente utilizadas com o objetivo de avaliar a mobilidade articular, planejar programas de intervençâo e traçar objetivos reais para cada indivíduo, especialmente com AR.

Historicamente, as avaliaçóes da ADM desenvolveram-se nos últimos 90 anos com o rápido desenvolvimento das ciências da saúde (GAJDOSIK; BOHANNON, 1987). A literatura descreve as medidas da amplitude desde a estimativa visual simples até as centrais inerciais e cinematografia de alta velocidade. Pela versatilidade, os instrumentos utilizados na prática clínica pelos fisioterapeutas são o goniômetro universal e o flexímetro pendular
(CAVE; ROBERTS, 1936; SILVA; IMOTO; CROCI, 2007; LIMA et al., 2004).

Nesta revisão bibliográfica, as formas de avaliação da ADM que mais predominaram foram a Goniometria (12 artigos), Escala da Escola Paulista de Medicina - Joint of Motion (EPM-ROM) (12 artigos) e Análise Cinemática (7 artigos), sendo que os demais artigos apresentavam protocolos de medida articular não comuns na prática clínica, como classificação por escores (HOOPER et al., 2014), teste funcional Keitel (POOLE et al., 2013), teste de mobilidade da mão (POOLE et al., 2013), "Working space of the hand" (DIAS et al., 2009), análise radiográfica (TÄGIL et al., 2009), teste do pêndulo (VALLE et al., 2006), índice de lesão articular (JOHNSON et al., 2002), estimativa visual (ORCES et al., 2002) e escala de movimento e alinhamento articular (CRANNEY et al., 1999).

A seguir, a descrição dos três principais protocolos de avaliação da ADM, que se destacaram na busca desta revisão.

\subsection{Goniometria}

Silva et al. (2011) relatam que até os dias atuais o instrumento mais utilizado para medida da ADM ainda é o goniômetro universal, como também corroborados por Batista et al. (2006), Sacco et al. (2007), Venturini et al. (2006), Norkin e White (2003) entre outros, mesmo quando comparado à goniometria radiológica, considerada referência ouro de amplitude real (SILVA et al., 2011). O goniômetro é basicamente um transferidor com dois braços longos, sendo que um braço é considerado móvel e o outro fixo, ambos presos ao corpo do transferidor por um rebite ou botão de tensão (PALMER; EPLER, 2000). Os mesmos autores referem que existem diversos estudos que comprovam a validade do goniômetro universal quando comparada à goniometria radiológica considerada referência de amplitude real. No entanto, a relativa morosidade para a aplicação dos testes goniométricos, que exigem certo grau de rigor e de experiência profissional, acaba por estimular o desuso desta técnica, popularizando procedimentos menos confiáveis, como a estimativa visual (MENADUE et al., 2006).

Nos estudos envolvendo a goniometria e AR, as articulaçōes investigadas foram: ombro (SLUNGAARD; MENGSHOEL, 2013; VERMEULEN et al., 2006), mão (FULFARO et al., 2012; HAYASHI; UCHIYA, 2011; NIEDERMANN et al., 2011; JOHNSSON; EBERHARDT, 2009; GOODSON et al., 2007; LEFEVRE-COLAU et al., 2003), punho (NIEDERMANN et al., 2011; JAIN et al., 2010) 
e joelho (NOLTE et al., 2011; MEIRELES et al., 2002). Apenas dois estudos avaliaram a ADM por meio exclusivamente goniométrico em pacientes com AR (ORCES et al., 2002; BADLEY; WAGSTAFF; WOOD, 1984).

Slungaard e Mengshoel (2013) avaliaram a flexão e abdução do ombro de forma ativa e passiva de acordo com as recomendaçóes propostas por Norkin e White (2003) e pela American Academy of Orthopedic Surgeons (AMERICAN..., 1965) e propuseram, como "posição zero" do ombro, o indivíduo sentado durante a abdução ativa e passiva e em supino durante a flexão ativa e passiva. A diferença entre abdução ativa e passiva e/ou entre a flexão ativa e passiva foi calculada e estimada como "déficit de movimento ativo", sendo estabelecido como ponto de corte $25^{\circ}$ da ADM normal (levando-se em consideração a possível margem de erro do goniômetro em $+/-5^{\circ}$ ). Da mesma forma, Vermeulen et al. (2006) utilizaram o protocolo da AAOS (AMERICAN..., 1965) com o mesmo nível de precisão $\left(5^{\circ}\right)$, e determinaram a função do ombro - ativa e passiva, em três movimentos: abdução, flexão e rotação externa. Entretanto, obtiveram todas as medidas na posição sentada.

Em relação às articulaçôes da mão reumatoide, a goniometria, assim como em outras articulaçóes acometidas, tem sido amplamente utilizada para avaliação de intervenção, como demonstrado no estudo de Niedermann et al. (2011) sobre medidas educacionais de proteção articular, em que se compararam dois protocolos e verificaram, entre outras medidas de capacidade funcional na AR, a $\mathrm{ADM}$ ativa da mão (movimentos de flexão e extensão das articulaçóes metacarpofalangeanas - MCF e punho dominante, sem descriçáo dos procedimentos de coleta da ADM). Da mesma forma, Hayashi e Uchiya (2011) utilizaram a goniometria para verificar formas de intervenção de splints de proteção articular de punho e mão em situação pré e pós-operatória e, neste caso, utilizaram um goniômetro para pequenas articulações e mediram a ADM das articulações MCF (flexão e extensão), posicionado no dorso das articulaçôes dos dedos, onde o arco de movimento ativo foi determinado subtraindo o valor da extensão ativa do valor da flexão ativa, corroborados por Goodson et al. (2007), acrescidos de que os ângulos articulares devem partir da posição de Zero Grau e que, na articulação do punho, o goniômetro apropriado é o universal.

Além destes estudos para avaliar a intervenção, LeFevre-Colau et al. (2003) propuseram um "Índice Modificado Kapandji” e utilizaram para construção deste índice a medida da mobilidade articular passiva em graus, por meio da goniometria, das articulaçôes
MCF e interfalangeanas proximais - IFP dos dedos e do polegar, bem como a articulação do punho (flexão e extensão, desvio ulnar e desvio radial) e articulação radioulnar (pronação e supinação). Em comum a este último estudo, há o fato de que, quando avaliada a função de punho, percebeu-se concomitantemente a medida da mobilidade radioulnar, como também citada no trabalho de Jain et al. (2010) sobre os efeitos da sinovectomia dos extensores e excisão da ulna distal em indivíduos com AR, provavelmente associada ao fato de que as funçóes plenas de mão e punho estejam diretamente relacionadas aos movimentos de pronação e supinação radioulnar.

Percebe-se, nos referidos estudos em indivíduos com AR que citam a avaliação da ADM, embora estejam relacionados à descriçáo da capacidade funcional ou como forma de intervenção de tratamento (cirúrgico, físico ou medicamentoso), que sempre estão associados à avaliação da funcionalidade e saúde geral, descritos no "Health Assessment Questionnaire" - HAQ, pois, juntos, fornecem informaçóes valiosas relacionadas à severidade da doença, como no estudo de Häkkinen et al. (2005b), que explorou a associação entre as subdimensões do HAQ e variáveis clínicas, em que quatro fisioterapeutas experientes mediram a ADM usando um goniômetro manual, com um nível de precisão de $5^{\circ}$, em posições padronizadas do "Zimmer Orthopaedic" das articulaçôes do cotovelo (flexão e déficit de extensão), punho (flexão e extensão) e joelho (flexão e déficit de extensão). Nas articulaçóes do cotovelo e joelho, a medida de extensão foi expressa em graus de déficit de extensão.

A medida da ADM é parâmetro determinante utilizado na avaliação e no acompanhamento fisioterapêutico e/ou de terapia ocupacional (VENTURINI et al., 2006). No caso da AR, pode ser uma ferramenta importante da definição da propedêutica e do prognóstico de um paciente submetido a tratamento conservador. O método de mensuração da ADM mais utilizado na prática clínica é a goniometria, com diferentes instrumentos para avaliar essas medidas como o goniômetro fluido, o eletrogoniômetro e o goniômetro universal (ALLINGER; ENGSBERG, 1993). Segundo Venturini et al. (2006), o goniômetro universal é de fácil aplicação, não invasivo, de baixo custo e, por isso, o mais utilizado na clínica fisioterapêutica. Entretanto, segundo os autores, a reprodutibilidade de suas medidas é mais limitada quando comparada a outros goniômetros, principalmente quando envolve diferentes examinadores, o que limita as reavaliaçóes periódicas que envolvam essas condiçôes. Além disso, a reprodutibilidade do goniômetro universal é examinador-dependente e varia de 
acordo com o nível de treinamento, com variaçóes entre $2^{\circ}$ e $7^{\circ}$ dentre as medidas consideradas aceitáveis, considerando as características da articulação a ser testada (BRAZ et al., 2008). Mesmo diante do exposto, Braz et al. (2008) destacam que, dentre as técnicas manuais de avaliaçáo, a goniometria é a que apresenta maior reprodutibilidade em mensuraçôes do arco, mas sua acurácia depende da habilidade e experiência do examinador.

\subsection{Escala da Escola Paulista de Medicina - Joint of Motion (EPM-ROM)}

Segundo Oliveira e Araújo (2006), a avaliação, em especial na AR, caminha cada vez mais para um procedimento que leve em conta a capacidade funcional, e a goniometria convencional, embora possa detectar a alteração da $\mathrm{ADM}$, não é capaz de mensurar de forma ideal alteraçôes na funcionalidade do indivíduo. Neste contexto, o protocolo proposto pela escala EPM-ROM (FERRAZ et al., 1990) utiliza a goniometria realizada por fisioterapeutas (OLIVEIRA et al., 2015; KUHLOW et al., 2010; BRODIN et al., 2008) e compara com valores considerados normais com o objetivo de detectar alteraçóes que ocorram ao longo da doença ou de uma intervenção terapêutica e possui, como vantagem, maior rapidez de avaliaçáo e menor manipulação do indivíduo, poupando-o de possíveis desconfortos.

A escala avalia dez movimentos distintos de pequenas e grandes articulaçóes e é baseada nos graus de movimento que são importantes na performance de atividades básicas da vida diária (BULTHUIS et al., 2007; VAN DEN ENDE et al., 2000). Os movimentos articulares incluídos no EPM-ROM, segundo Van den Ende et al. (1996), são: flexão e extensão de cotovelo, flexão e extensão de punho, a média da flexão das articulaçóes metacarpofalangeanas, abdução do polegar, flexão da articulação interfalangeana proximal do polegar, flexão de quadril, extensão de joelho e flexão plantar do tornozelo; os movimentos são registrados em graus $\left({ }^{\circ}\right)$ e os posicionamentos estão em acordo com medidas ortopédicas padróes da AAOS - American Academy of Orthopaedic Surgeons.

A avaliação é realizada de forma ativa-assistida, em que o indivíduo realiza o movimento e a mão do avaliador acompanha o movimento nos últimos graus de ADM. A escala divide-se em quatro faixas de ADM, em que " 0 " significa movimento normal (nenhuma incapacidade articular); "1" demonstra alguma perda de capacidade funcional, porém a articulação ainda colabora (incapacidade articular leve); "2", a perda é considerável e obriga outras articulaçóes a suprirem a falta de ADM principal (incapacidade articular moderada); "3", alto nível de deterioração, difícil de recuperar a articulação sem procedimento cirúrgico (incapacidade articular severa) (VAN DEN ENDE et al., 1996; FERRAZ et al., 1990).

A nota da escala, que vai de "0 a 30 ", é dada pela soma de notas de um movimento em que a faixa " 0 " soma " 0 " pontos, a faixa "1" soma " 1 " ponto, a faixa " 2 " soma " 2 " pontos e a faixa " 3 " soma " 3 " pontos. A nota é dividida entre lado direito e esquerdo e a nota final é a soma das notas de todos os movimentos; em que "0" corresponde à ausência de restrição do movimento e, quanto maior a nota, pior o estado funcional do paciente (BRODIN et al., 2008; BULTHUIS et al., 2007; OLIVEIRA; ARAÚJO, 2006).

Dentre os artigos levantados sobre avaliação funcional versus $A D M$ na $A R$, destacaram-se os relacionados ao EPM-ROM (12 artigos). Destes, cinco trabalhos o utilizaram como forma de medida em intervençóes de terapia por meio de exercícios, como se verificou nos trabalhos de Brodin et al. (2008), Oosterveld et al. (2009), Bulthuis et al. (2007) e Van den Ende et al. (2000, 1996). Em comum, observa-se um bom número de participantes: 228,38 , 98, 64 e 100, respectivamente, levando-se em conta a estimativa de que a AR ocorra entre 0,5 e $1 \%$ da população mundial, ou seja, o acesso é restrito a esses pacientes, por dificuldade ao acesso ao diagnóstico e baixa prevalência quando comparada a outras enfermidades. Além disso, esses estudos faziam avaliaçóes da ADM no decorrer da intervenção.

Em relação ao acompanhamento funcional (OLIVEIRA et al., 2015; KUHLOW et al., 2010), Kuhlow et al. (2010) descrevem o EPM-ROM como uma medida padronizada da amplitude potencial do movimento das articulações em membros superiores e inferiores e, de acordo com Oliveira et al. (2015), em conjunto com o HAQ - Health Assessment Questionnaire, podem refletir a alteração da capacidade funcional ao longo do tempo, como parâmetro de resultado da indicação de cirurgia ortopédica. Os estudos de Cranney et al. (1999) e Pincus et al. (1997) corroboram esses achados e afirmam por seus estudos que escalas de deformidade e limitação de movimento articular são fortemente correlacionadas com escores radiográficos na AR crônica e defendidas como medidas de lesão articular total.

Kuhlow et al. (2010), em um estudo transversal com 239 pacientes em dois centros (Zurique e Munique), para explorar as limitaçóes na atividade de acordo com a CIF - Classificação Internacional de 
Funcionalidade, avaliaram a ADM pelo EPM-ROM simultâneo a outras variáveis como HAQ, o DAS-28 - Disease Activity Score, RADAI - Rheumatoid Arthritis Disease Activity Index, SF-36 - Short-Form Health Survey, SODA - Sequentional Occupational Dexterity Assessment, MSI - Muscle Strenght Index, Ratingen Score e questionários sociodemográficos e de comorbidades e constataram que a ADM e a força muscular são parâmetros importantes para a reabilitação e a prática clínica.

Os demais estudos desta revisão relatavam a avaliação em um corte transversal entre pacientes com diagnóstico de AR e pacientes controles saudáveis (OLIVEIRA et al., 2014); avaliação isocinética do joelho, associada à avaliação da ADM e HAQ (MEIRELES et al., 2002); criação de um Índice de Deformidade baseado na junção de duas escalas Joint Alignment and Motion Scale e Escola Paulista de Medicina - Range of Motion (CRANNEY et al., 1999), uma revisão de literatura sobre métodos de avaliação na AR (STENSTRÖM; NISELL, 1997), uma investigaçáo sobre a confiabilidade inter e intraobservador (VLIELAND et al., 1993) e o estudo que lançou a escala em 1990 (FERRAZ et al., 1990).

\subsection{Análise cinemática}

Consiste no registro de imagens captadas por câmeras e auxílio de correspondentes "Soft"- e "Hardware" e as consequentes reconstruçôes com auxílio de pontos marcados, conforme modelo antropométrico, que estima a localização dos eixos articulares do sujeito e onde se fixam estas marcas anatômicas e as coordenadas tridimensionais de cada ponto corporal para cada quadro, dentro do espectro de frequência do registro, serão determinadas através desse ponto juntamente com as funçôes trigonométricas e de cálculos de variáveis cinemáticas (AMADIO; SERRÁO, 2007). Apresenta seis graus de liberdade pelos ângulos de Euler, com sequências de Pitch (x: translação frontal), Yaw (y: translação sagital) e Roll (z: translaçáo axial), ou seja, dois sistemas de coordenadas - um sistema inercial fixo e outro que gira junto ao corpo em rotação e que especifica a orientação do corpo girante em relação ao sistema inercial (fixo) (SUGIURA et al., 2014).

De acordo com Lima et al. (2008), a análise cinemática do movimento humano tem sido utilizada como método de avaliação quantitativa, a qual, por meio da interpretaçáo dos resultados, permite a inferência sobre detalhes do movimento. Tal análise tem sido amplamente empregada nas diferentes áreas de estudo da motricidade humana, seja para avaliação do desempenho de atletas nos esportes e dos efeitos da reabilitação, seja para ampliar os conhecimentos sobre desenvolvimento e controle motor em crianças e adultos.

$\mathrm{Na}$ avaliação da coluna cervical, os dois artigos analisados utilizaram modelo tridimensional obtido a partir da Tomografia Computadoriza (TC). Takatori et al. (2010) analisaram as alteraçóes morfológicas e cinemáticas da cervical de indivíduos com $\mathrm{AR}$, através de medidas seriadas de $\mathrm{TC}$ da regiáo cervical em posição neutra e flexionada. Os autores criaram um modelo tridimensional através do método de volume de mesclagem e, a partir deste, mensuraram a flexão, extensão e rotaçáo da cervical.

Já Sugiura et al. (2014) analisaram as alteraçôes morfológicas e cinemáticas da cervical superior em indivíduos com AR e para tal utilizaram TC seriada com a cervical em posição neutra e com rotaçáo em $45^{\circ}$ para a direita e para a esquerda e, através de uma análise matemática complexa, obtiveram um modelo cinemático 3D. Os autores apontaram algumas limitaçóes do estudo como: a) as imagens náo foram obtidas em tempo real e na posiçấo vertical; b) o aumento do movimento segmentar causado pela frouxidão ligamentar e ruptura do ligamento cruzado náo poderia ser detectado porque o estudo foi realizado com a cabeça fixada apenas com $45^{\circ} \mathrm{de}$ rotação; c) a exigência de pacientes que alcançavam $45^{\circ}$ graus de rotação de cervical afastou do estudo pacientes com doença mais severa.

Weiss et al. (2007) e Dubbeldam et al. (2013, 2011) analisaram o movimento articular durante o ciclo da marcha. Desta forma, Weiss et al. (2007) observaram as alteraçóes da mobilidade articular e seus resultados funcionais após artrodese de retropé em indivíduos com AR durante a marcha em velocidade espontânea. Para tal, utilizaram-se 34 marcadores reflexivos em pontos ósseos padronizados e 6 câmeras para criar um modelo tridimensional através do Vicon Moviments System. Os autores ponderaram como limitação que o modelo tridimensional, criado no estudo, analisou o pé como um único segmento, impossibilitando uma análise mais refinada.

Dubbeldam et al. (2011) avaliaram o efeito da velocidade da marcha nas características cinemáticas em indivíduos com AR. Os autores também utilizaram o Vicon Moviments System, entretanto, optaram por 19 marcadores no membro inferior avaliado e a análise da marcha em velocidades pré-determinadas e não relataram, ao longo do estudo, nenhuma dificuldade ou limitação neste método de avaliação.

Já no estudo de 2013, Dubbeldam et al. avaliaram o efeito das alteraçóes clínicas e morfológicas do dano articular do pé e tornozelo em pacientes com 
AR, na cinemática da marcha. Pode-se salientar que o diferencial deste estudo foi a utilização de um modelo de análise mais refinado, que permitiu a avaliação do antepé, mediopé e retropé durante os ciclos da marcha.

Em relação à análise cinemática das mãos, apesar da predileção desta articulação na AR, encontraram-se apenas dois estudos na temática envolvendo análise de ADM, a saber: Fowler e Nicol (2001) e Lester et al. (2012).

Fowler e Nicol (2001) avaliaram a mobilidade da mão durante a realização de testes funcionais, amplamente utilizados para avaliar a funcionalidade na mão reumatoide. Os autores utilizaram 4 marcadores reflexivos na mão e 6 câmeras para criar um modelo tridimensional através do Vicon Moviments System. Entretanto, o diferencial deste estudo foi a análise do movimento em relação à função, desta forma, os marcadores reflexivos foram colocados em pontos pré-definidos e acoplados de 3 mini-hastes representando os sistemas de eixos ortogonais. Assim, os autores obtiveram modelo tridimensional do movimento durante atividades funcionais.

Já o estudo de Lester et al. (2012) descreve detalhadamente $\mathrm{o}$ arco de movimento da articulaçáo metacarpofalangeana em indivíduos com e sem substituição articular. Os autores utilizaram 34 marcadores reflexivos na máo e 12 câmeras para criar um modelo tridimensional através do Vicon Moviments System. A ADM foi analisada durante o movimento de pinça e preensão da mão, abdução/adução, flexão/extensão dos dedos. Os autores relataram como limitação da cinemática em pequenas articulaçóes a sobreposição de imagens, o que provoca lacunas de dados, que, neste estudo, foi corrigida automaticamente nas pequenas lacunas e através do software da própria Vicon nas lacunas maiores.

\section{Conclusão}

Em relação aos protocolos de avaliação da ADM utilizando a goniometria, assim como nos estudos que utilizaram a escala EPM-ROM, poucos se preocuparam em descrever a forma de aquisição das medidas, impossibilitando a reprodução das técnicas de medida na AR.

É de suma importância a familiarização dos métodos pela equipe multidisciplinar que acompanha o paciente com AR, de forma a viabilizar uma avaliação que relacione efeitos de intervençóes - sejam elas medicamentosas ou não, para a melhora da qualidade de vida do indivíduo com complicaçóes decorrentes da AR.
Como se percebe, os estudos náo trazem a ênfase ao estudo da mobilidade articular de indivíduos com AR, sendo comum a inclusão da avaliação da ADM como uma maneira de acompanhar a evoluçáo da doença diretamente à função das articulaçôes, principalmente as mais comprometidas. Portanto, após a análise dos artigos expostos, sugere-se o estudo completo do comprometimento articular com o objetivo de traçar propósitos de intervençáo ou prevenção de deformidades geradas pela AR e que comprometem drasticamente a qualidade de vida destes pacientes em suas atividades de vida diária - sejam elas laborais, domésticas ou de lazer - e, acima de tudo, difundir o uso de tais práticas de avaliaçáo entre a equipe multidisciplinar que acompanha este perfil, em especial fisioterapeutas, terapeutas ocupacionais, educadores físicos e médicos, a fim de otimizar o plano de assistência a longo prazo, levando-se em conta a cronicidade da condição.

\section{Referências}

ALETAHA, D. et al. Rheumatoid arthritis classification criteria. Arthritis \& Rheumatism, Malden, v. 62, n. 9, p. 2569-2581, 2010. PMid:20872595. http://dx.doi. org/10.1002/art.27584.

ALLINGER, T. L.; ENGSBERG, J. R. A method to determine the range of motion of the ankle joint complex, in vivo. Journal of Biomechanics, Philadelphia, v. 26, n. 1, p. 69-76, 1993. PMid:8423170. http://dx.doi. org/10.1016/0021-9290(93)90614-K.

ALVES, L. C. et al. A influência das doenças crônicas na capacidade funcional dos idosos do município de São Paulo, Brasil. Cadernos de Saúde Pública, Rio de Janeiro, v. 23, n. 8, p. 1924-1930, 2007.

AMADIO, A. C.; SERRÃO, J. C. Contextualização da biomecânica para a investigação do movimento: fundamentos, métodos e aplicaçôes para análise da técnica esportiva. Revista Brasileira de Educação Física e Esporte, São Paulo, v. 21, p. 61-85, 2007. Número Especial.

AMERICAN ACADEMY OF ORTHOPAEDIC SURGEONS. Joint motions: method of measuring and recording. Joint motion method of measuring and recording. Chicago, 1965.

BADLEY, E. M.; WAGSTAFF, S.; WOOD, P. H. Measures of functional ability (disability) in arthritis in relation to impairment of range of joint movement. Annals of the Rheumatic Diseases, London, v. 43, n. 4, p. 563569, 1984. PMid:6236760. http://dx.doi.org/10.1136/ $\operatorname{ard} .43 .4 .563$.

BATISTA, L. H. et al. Avaliação da amplitude articular do joelho: correlaçáo entre as medidas realizadas com o goniômetro universal e no dinamômetro isocinético. Brazilian Journal of Physical Therapy, São Carlos, v. 10, n. 2, p. 193-198, 2006. 
BENDTSEN, P. et al. Cross-sectional assessment and subgroup comparison of functional disability in patients with rheumatoid arthritis in a Swedish health-care district. Disability and Rehabilitation, Oxfordshire, v. 17, n. 2, p. 94-99, 1995. PMid:7795266. http://dx.doi. org/10.3109/09638289509166634.

BERTOLO, M. B. Como diagnosticar e tratar artrite reumatóide. Revista Brasileira de Medicina, São Paulo, v. 65, n. 12, p. 64-72, 2008.

BRANDÃO, L. Avaliação da qualidade de vida na artrite reumatóide: revisão atualizada. Revista Brasileira de Reumatologia, Sáo Paulo, v. 37, n. 5, p. 275-281, 1997.

BRAZ, R. G. et al. Confiabilidade e validade de medidas angulares por meio do software para avaliaçáo postural. Fisioterapia em Movimento, Curitiba, v. 21, n. 3, p. 117 126, 2008.

BRODIN, N. et al. Coaching patients with early rheumatoid arthritis to healthy physical activity: a multicenter, randomized, controlled study. Arthritis and Rheumatism, Malden, v. 59, n. 3, p. 325-331, 2008. PMid:18311770.

BULTHUIS, Y. et al. Arthritis patients show long-term benefits from 3 weeks intensive exercise training directly following hospital discharge. Rheumatology, Oxford, v. 46, n. 11, p. 1712-1717, 2007. PMid:17956917. http:// dx.doi.org/10.1093/rheumatology/kem236.

CAVE, E. F; ROBERTS, S. M. A method for measuring and recording joint function. Journal of Bone and Joint Surgery, Boston, v. 18, n. 2, p. 455-465, 1936.

COORDENAÇÃO DE APERFEIÇOAMENTO DE PESSOAL DE NÍVEL SUPERIOR. Brasília, 2016. Disponível em: <http://www.periodicos.capes.gov.br/>. Acesso em: 26 jan. 2016.

CORBACHO, M. I.; DAPUETO, J. J. Avaliação da capacidade funcional e da qualidade de vida de pacientes com artrite reumatóide. Revista Brasileira de Reumatologia, São Paulo, v. 50, n. 1, p. 31-43, 2010. http://dx.doi. org/10.1590/S0482-50042010000100004.

CRANNEY, A. et al. A measure of limited joint motion and deformity correlates with HLA-DRB1 and DQB1 alleles in patients with rheumatoid arthritis. Annals of the Rheumatic Diseases, London, v. 58, n. 11, p. 703-708, 1999. PMid:10531075. http://dx.doi.org/10.1136/ ard.58.11.703.

DIAS, J. J. et al. The working space of the hand in rheumatoid arthritis: its impact on disability. The Journal of Hand Surgery, London, v. 34, n. 4, p. 465-470, 2009. PMid:19474183. http://dx.doi. org/10.1177/1753193409103244.

DUARTE, Y. A. O.; ANDRADE, C. L.; LEBRÃO, M. L. O Índex de Katz na avaliação da funcionalidade dos idosos. Revista da Escola de Enfermagem da USP, São Paulo, v. 41, n. 2, p. 317-325, 2007. http://dx.doi. org/10.1590/S0080-62342007000200021.
DUBBELDAM, R. et al. Foot and ankle joint kinematics in rheumatoid arthritis cannot only be explained by alteration in walking speed. Gait \& Posture, Philadelphia, v. 33, n. 3, p. 390-395, 2011. PMid:21295983. http://dx.doi.org/10.1016/j.gaitpost.2010.12.010.

DUBBELDAM, R. et al. Foot and ankle kinematics in rheumatoid arthritis: influence of foot and ankle joint and leg tendon pathologies. Arthritis Care \& Research, Atlanta, v. 65, n. 4, p. 503-511, 2013. PMid:22972768. http://dx.doi.org/10.1002/acr.21852.

ENOKA, R. M. Bases neuromecânicas da cinesiologia. São Paulo: Manole, 1999.

FALEIRO, L. R. et al. A Terapia Anti-TNF- $\alpha$ na Artrite Reumatóide. Semina: Ciências Biológicas e da Saúde, Londrina, v. 32, n. 1, p. 77-94, 2011.

FERRAZ, M. B. et al. EPM-ROM Scale: an evaluative instrument to be used in rheumatoid arthritis trials. Clinical and Experimental Rheumatology, Oxford, v. 8, n. 5, p. 491-494, 1990.

FLOYD, R. T. Manual de cinesiologia estrutural. Barueri: Manole, 2011.

FOWLER, N. K.; NICOL, A. C. Functional and biomechanical assessment of the normal and rheumatoid hand. Clinical Biomechanics, Pennsylvania, v. 16, n. 8, p. 660-666, 2001. PMid:11535347. http://dx.doi. org/10.1016/S0268-0033(01)00057-2.

FULFARO, M. A. et al. Caracterização funcional de idosos com artrite reumatóide. Estudos Interdisciplinares sobre o Envelhecimento, Porto Alegre, v. 17, n. 2, p. $305-$ 319, 2012.

GAJDOSIK, R. L.; BOHANNON, R. W. Clinical measurement of range of motion. Review of goniometry emphasizing reliability and validity. Physical Therapy, Alexandria, v. 67, n. 12, p. 1867-1872, 1987. PMid:3685114. http://dx.doi.org/10.1093/ptj/67.12.1867.

GOODSON, A. et al. Direct, quantitative clinical assessment of hand function: usefulness and reproducibility. Manual Therapy, Philadelphia, v. 12, n. 2, p. 144-152, 2007. PMid:16899386. http://dx.doi.org/10.1016/j. math.2006.06.002.

HÄKKINEN, A. et al. Decreased muscle strength and mobility of the neck in patients with rheumatoid arthritis and atlantoaxial disorders. Archives of Physical Medicine and Rehabilitation, Philadelphia, v. 86, n. 8, p. 1603-1608, 2005a. PMid:16084814. http://dx.doi. org/10.1016/j.apmr.2005.02.011.

HÄKKINEN, A. et al. Pain and joint mobility explain individual subdimensions of the health assessment questionnaire (HAQ) disability index in patients with rheumatoid arthritis. Annals of the Rheumatic Diseases, London, v. 64, n. 1, p. 59-63, 2005b. PMid:15130901. http://dx.doi.org/10.1136/ard.2003.019935.

HAMBRIGHT, D. et al. A comparison of perioperative outcomes in patients with and without rheumatoid 
arthritis after receiving a total shoulder replacement arthroplasty. Journal of Shoulder and Elbow Surgery, Philadelphia, v. 20, n. 1, p. 77-85, 2011. PMid:20655764.

HAMILL, J.; KNUTZEN, K. M. Bases biomecânicas do movimento humano. São Paulo: Manole, 1999.

HAMMOND, A.; FREEMAN, K. One-year outcomes of a randomized controlled trial of an educational-behavioural joint protection programme for people with rheumatoid arthritis. Rheumatology, Oxford, v. 40, n. 9, p. 1044-1051, 2001. PMid:11561117. http://dx.doi. org/10.1093/rheumatology/40.9.1044.

HAYASHI, H.; UCHIYA, J. A 3-year Follow-up Study on the Alternating Use of Static Splints after Metacarpophalangeal Joint Arthroplasty in a Patient with Rheumatoid Arthritis. Asian Journal of Occupational Therapy, Tóquio, v. 9, n. 1, p. 1-5, 2011. http://dx.doi. org/10.11596/asiajot.9.1.

HOOPER, L. et al. Comparative distribution of ultrasound-detectable forefoot bursae in patients with osteoarthritis and rheumatoid arthritis. Arthritis Care \& Research, Atlanta, v. 66, n. 6, p. 869-877, 2014. http:// dx.doi.org/10.1002/acr.22217.

JAIN, A. et al. Effects of extensor synovectomy and excision of the distal ulna in rheumatoid arthritis on long-term function. The Journal of Hand Surgery, Philadelphia, v. 35, n. 9, p. 1442-1448, 2010. PMid:20673615. http://dx.doi.org/10.1016/j.jhsa.2010.04.034.

JOHNSON, A. H. et al. The mechanical joint score: a new clinical index of joint damage in rheumatoid arthritis. Rheumatology, Oxford, v. 41, n. 2, p. 189-195, 2002. PMid:11886969. http://dx.doi.org/10.1093/rheumato$\log y / 41.2 .189$.

JOHNSSON, P. M.; EBERHARDT, K. Hand deformities are important signs of disease severity in patients with early rheumatoid arthritis. Rheumatology, Oxford, v. 48, n. 11, p. 1398-1401, 2009. PMid:19720679. http://dx.doi.org/10.1093/rheumatology/kep253.

KENDALL, F.; MCCREARY, E. Músculos: provas e funçôes. São Paulo: Manole, 1990.

KUHLOW, H. et al. Factors explaining limitations in activities and restrictions in participation in rheumatoid arthritis. European Journal of Physical and Rehabilitation Medicine, Torino, v. 46, n. 2, p. 169-177, 2010. PMid:20485222.

LAMB, S. E. et al. Exercises to improve function of the rheumatoid hand (SARAH): a randomised controlled trial. The Lancet, Philadelphia, v. 385, n. 9966, p. 421-429, 2015. PMid:25308290. http://dx.doi. org/10.1016/S0140-6736(14)60998-3.

LEFEVRE-COLAU, M. M. et al. Reliability, validity, and responsiveness of the modified Kapandji index for assessment of functional mobility of the rheumatoid hand. Archives of Physical Medicine and Rehabilitation, Philadelphia, v. 84, n. 7, p. 1032-1038, 2003.
PMid:12881830. http://dx.doi.org/10.1016/S0003-9993(03)00128-X.

LESTER, L. E. et al. Range of motion of the metacarpophalangeal joint in rheumatoid patients, with and without a flexible joint replacement prosthesis, compared with normal subjects. Clinical Biomechanics, Philadelphia, v. 27, n. 5, p. 449-452, 2012. PMid:22261011. http://dx.doi.org/10.1016/j.clinbiomech.2011.12.010.

LIMA, C. D. et al. Dois métodos diferentes para análise cinemática dos movimentos de cabeça durante a coordenação viso-cefálica de lactentes. Brazilian Journal of Physical Therapy, São Carlos, v. 12, n. 5, p. 425-431, 2008. http://dx.doi.org/10.1590/S141335552008000500013 .

LIMA, L. A. O. et al. Estudo da confiabilidade de um instrumento de medida de flexibilidade em adultos e idosos. Revista de Fisioterapia da Universidade de São Paulo, São Paulo, v. 11, n. 2, p. 83-89, 2004.

MARQUES NETO, J. F. et al. Estudo multicêntrico da prevalência da artrite reumatóide do adulto em amostras da população brasileira. Revista Brasileira de Reumatologia, São Paulo, v. 33, n. 5, p. 169-173, 1993.

MASANES, F. et al. Prevalence of sarcopenia in healthy community-dwelling elderly in an urban area of Barcelona (Spain). The Journal of Nutrition, Health \& Aging, Oxford, v. 16, n. 2, p. 184-187, 2012. PMid:22323356. http://dx.doi.org/10.1007/s12603-011-0108-3.

MEIRELES, S. M. et al. Isokinetic evaluation of the knee in patients with rheumatoid arthritis. Joint Bone Spine, Philadelphia, v. 69, n. 6, p. 566-573, 2002. PMid:12537264. http://dx.doi.org/10.1016/S1297$-319 X(02) 00453-0$.

MENADUE, C. et al. Reliability of two goniometric methods of measuring active inversion and eversion range of motion at the ankle. BMC Musculoskeletal Disorders, London, v. 7, n. 1, p. 60-67, 2006. PMid:16872545. http://dx.doi.org/10.1186/1471-2474-7-60.

NIEDERMANN, K. et al. Effectiveness of individual resource-oriented joint protection education in people with rheumatoid arthritis: a randomized controlled trial. Patient Education and Counseling, Philadelphia, v. 82, n. 1, p. 42-48, 2011. PMid:20451345. http://dx.doi. org/10.1016/j.pec.2010.02.014.

NISHIKAWA, M. et al. Acquired permanent dislocation of the patella in a patient with rheumatoid genu valgum. Journal of Clinical Orthopaedics and Trauma, Philadelphia, v. 6, n. 2, p. 120-125, 2015. PMid:25983519. http://dx.doi.org/10.1016/j.jcot.2015.01.094.

NOLTE, K. et al. Land- and water-based exercises in rheumatoid arthritis patients: a series of case reports. South African Journal of Sports Medicine, Rondebosch, v. 23, n. 3, p. 84-88, 2011.

NORKIN, C. C.; WHITE, D. J. Measurement of joint motion: a guide to goniometry. Philadelphia: F. A. Davis Company, 2003. 
OLIVEIRA, L. M. et al. Acompanhamento da capacidade funcional de pacientes com artrite reumatoide por três anos. Revista Brasileira de Reumatologia, São Paulo, v. 55, n. 1, p. 62-67, 2015.

OLIVEIRA, L. M.; ARAÚJO, P. M. P. A. Manual de medida articular. São Paulo: Editora Atheneu, 2006.

OLIVEIRA, S. C. G. et al. Avaliaçâo isocinética do tornozelo de pacientes com artrite reumatóide. Revista Brasileira de Reumatologia, São Paulo, v. 55, n. 4, p. 318324, 2014.

OOSTERVELD, F. G. J. et al. Infrared sauna in patients with rheumatoid arthritis and ankylosing spondytlitis. Clinical Rheumatology, Oxford, v. 28, n. 1, p. 29-34, 2009. http://dx.doi.org/10.1007/s10067-008-0977-y.

ORCES, C. H. et al. The number of deformed joints as a surrogate measure of damage in rheumatoid arthritis. Arthritis and Rheumatism, Malden, v. 47, n. 1, p. 67-72, 2002.

PALMER, L. M.; EPLER, M. E. Fundamentos das técnicas de avaliação musculoesquelética. Rio de Janeiro: Guanabara Koogan, 2000.

PINCUS, T. et al. Comparison of 3 quantitative measures of hand radiographs in patients with reumatoid arthritis: Steinbrocker Stage, Kaye modified Sharp score, and Larsen score. Journal of Rheumatology, Toronto, v. 24, n. 11, p. 2106-2112, 1997. PMid:9375867.

POOLE, J. L.; SANTHANAM, D. D.; LATHAM, A. L. Hand impairment and activity limitations in four chronic diseases. Journal of Hand Therapy, Philadelphia, v. 26, n. 3, p. 232-236, quiz 237, 2013. PMid:23622819. http://dx.doi.org/10.1016/j.jht.2013.03.002.

SACCO, I. C. N. et al. Confiabilidade da fotogrametria em relação a goniometria para avaliação postural de membros inferiores. Brazilian Journal of Physical Therapy, São Carlos, v. 11, n. 5, p. 411-417, 2007.

SANTANA, F. S. et al. Avaliação da capacidade funcional em pacientes com artrite reumatóide: implicaçôes para a recomendação de exercícios físicos. Revista Brasileira de Reumatologia, São Paulo, v. 54, n. 5, p. 378-385, 2014.

SENNA, E. R. et al. Prevalence of rheumatic diseases in Brazil: a study using the COPCORD approach. The Journal of Rheumatology, Toronto, v. 31, n. 3, p. 594597, 2004. PMid:14994410.

SILVA, A. L. P.; IMOTO, D. M.; CROCI, A. T. Estudo comparativo entre a aplicação de crioterapia, cinesioterapia e ondas curtas no tratamento da osteoartrite de joelho. Acta Ortopédica Brasileira, São Paulo, v. 15, n. 4, p. 204-209, 2007.

SILVA, E. et al. Declining use of orthopedic surgery in patients with rheumatoid arthritis? Results of a long-term, population-based assessment. Arthritis and
Rheumatism, Malden, v. 49, n. 2, p. 216-220, 2003. PMid:12687513. http://dx.doi.org/10.1002/art.10998.

SILVA, R. L. F. et al. Correlação entre fleximetria e goniometria radiológica para avaliaçóes da amplitude articular estática do cotovelo. Fisioterapia Brasil, São Paulo, v.12, n. 5, p. 359-364, 2011.

SLUNGAARD, B.; MENGSHOEL, A. M. Shoulder function and active motion deficit in patients with rheumatoid arthritis. Disability and Rehabilitation, London, v. 35 , n. 16, p. 1357-1363, 2013. PMid:23116340. http://dx.doi.org/10.3109/09638288.2012.732187.

STENSTRÖM, C. H.; NISELL, R. Assessment of disease consequences in rheumatoid arthritis: a survey of methods classified according to the International Classification of Impairments, Disabilities, and Handicaps. Arthritis Care and Research, Atlanta, v. 10, n. 2, p. 135150, 1997. PMid:9313402.http://dx.doi.org/10.1002/ art.1790100209.

SUGIURA, T. et al. In vivo 3D kinematics of the upper cervical spine during head rotation in rheumatoid arthritis. Journal of Neurosurgery: Spine, Charlottesville, v. 20, n. 4, p. 404-410, 2014. PMid:24506101.

SWEENEY, S. E.; FIRESTEIN, G. S. Rheumatoid arthritis: regulation of synovial inflammation. The International Journal of Biochemistry \& Cell Biology, Amsterdam, v. 36, n. 3, p. 372-378, 2004. PMid:14687914. http:// dx.doi.org/10.1016/S1357-2725(03)00259-0.

TÄGIL, M. et al. Correlation between range of motion and implant fracture: a 5 year follow-up of 72 joints in 18 patients in a randomized study comparing Swanson and Avanta/Sutter MCP silicone prosthesis. The Journal of Hand Surgery, London, v. 34, n. 6, p. 743-747, 2009. PMid:19786405. http://dx.doi. org/10.1177/1753193409346793.

TAKATORI, R. et al. Three-dimensional morphology and kinematics of the craniovertebral junction in rheumatoid arthritis. Spine, Hagerstown, v. 35, n. 23, p. 1278-1284, 2010. PMid:20736886. http://dx.doi. org/10.1097/BRS.0b013e3181e6d578.

TORQUETTI, A. et al. Programas de proteçáo articular para indivíduos com artrite reumatóide: uma revisão da literatura. Revista Terapia Ocupacional Universidade de São Paulo, São Paulo, v. 19, n. 2, p. 76-84, 2008.

VALLE, M. S. et al. The pendulum test as a tool to evaluate passive knee stiffness and viscosity of patients with rheumatoid arthritis. BMC Musculoskeletal Disorders, London, v. 7, n. 89, p. 1-12, 2006. PMid:17134492.

VAN DEN ENDE, C. H. M. et al. Comparison of high and low intensity training in well controlled rheumatoid arthritis: results of a randomised clinical trial. Annals of the Rheumatic Diseases, London, v. 59, n. 8, p. 615-621, 2000. http://dx.doi.org/10.1136/ard.59.8.615.

VAN DEN ENDE, C. H. M. et al. Effect of intensive exercise on patients with active rheumatoid arthritis: a randomised clinical trial. Annals of the Rheumatic Disea- 
ses, London, v. 55, n. 8, p. 798-805, 1996. http://dx.doi. org/10.1136/ard.55.11.798.

VENTURINI, C. et al. Confiabilidade intra e interexaminadores de dois métodos de medida da amplitude ativa de dorsiflexão do tornozelo em indivíduos saudáveis. Brazilian Journal of Physical Therapy, São Carlos, v. 10, n. 4, p. 407-411, 2006.

VERMEULEN, H. M. et al. Responsiveness of the shoulder function assessment scale in patients with rheumatoid arthritis. Annals of the Rheumatic Diseases, Lon- don, v. 65, n. 2, p. 239-241, 2006. PMid:16410527. http://dx.doi.org/10.1136/ard.2005.036236.

VLIELAND, T. P. M. V. et al. Evaluation of joint mobility in rheumatoid arthritis trials: the value of the EPM-range of motion scale. The Journal of Rheumatology, Toronto, v. 20, n. 12, p. 2010-2014, 1993. PMid:8014926.

WEISS, R. J. et al. Ankle/hindfoot arthrodesis in rheumatoid arthritis improves kinematics and kinetics of the knee and hip: a prospective gait analysis study. Rheumatology, Oxford, v. 46, p. 1024-1028, 2007.

\section{Contribuição dos Autores}

Melissa Andrea Jeannet Michaelsen Cardoso Mezzari, Juliane de Oliveira, Susana Cristina Domenech, Noé Gomes Borges Júnior e Monique da Silva Gevaerd contribuíram igualmente na concepção do artigo. Todos os autores aprovaram a versão final do texto. 